\title{
Dark Matter and Dark Energy Induced by Condensates
}

\author{
Antonio Capolupo \\ Dipartimento di Fisica E.R. Caianiello and INFN Gruppo Collegato di Salerno, Universitá di Salerno, 84084 Fisciano, Italy
}

Correspondence should be addressed to Antonio Capolupo; capolupo@sa.infn.it

Received 3 May 2016; Revised 23 July 2016; Accepted 18 August 2016

Academic Editor: Juan José Sanz-Cillero

Copyright (C) 2016 Antonio Capolupo. This is an open access article distributed under the Creative Commons Attribution License, which permits unrestricted use, distribution, and reproduction in any medium, provided the original work is properly cited. The publication of this article was funded by SCOAP ${ }^{3}$.

It is shown that the vacuum condensate induced by many phenomena behaves as a perfect fluid which, under particular conditions, has zero or negative pressure. In particular, the condensates of thermal states of fields in curved space and of mixed particles have been analyzed. It is shown that the thermal states with the cosmic microwave radiation temperature and the Unruh and the Hawking radiations give negligible contributions to the critical energy density of the universe, while the thermal vacuum of the intercluster medium could contribute to the dark matter, together with the vacuum energy of fields in curved space-time and of mixed neutrinos. Moreover, a component of the dark energy can be represented by the vacuum of axion-like particles mixed with photons and superpartners of neutrinos. The formal analogy among the systems characterized by the condensates can open new scenarios in the possibility of detecting the dark components of the universe in table top experiments.

\section{Introduction}

Many independent experimental data [1-5] support the hypothesis according to which the today observed universe has an accelerating expansion due to an unknown form of energy, called dark energy, which has a negative pressure. The dark energy represents approximately $68 \%$ of the total matter-energy density of the universe and it is distributed isotropically throughout the universe. Moreover, astronomical observations relative to the speed of rotating galaxies indicate that an unknown form of matter, not interacting electromagnetically, is needed in order to permit the stability of the galaxies and of cluster of galaxies. The dark matter makes up about $27 \%$ of the universe. Different models have been proposed to solve the dark energy [6-25] and dark matter puzzles [26-32]; however, the explanation of the dark components of the universe represents still a very big challenge.

Apparently separate research lines regard the study of physical systems characterized by vacuum condensates [3350], such as the Hawking or the Casimir effect $[39,40]$. Such phenomena have a nonzero vacuum energy which cannot be removed by use of the normal ordering procedure. This fact, in a supersymmetric context, induces the spontaneous breaking of supersymmetry [51-56].

In the present paper it is shown that these interesting issues are intimately bound together in such way that the vacuum condensate energy can provide contributions to the dark energy and to the dark matter. It is shown that all the condensates have state equations depending on the particular regime one considers (high momentum (UV) and low momentum (IR) regime). The particular cases of the vacuum energy induced by thermal states by curved spaces and by the mixing of particles are analyzed in detail.

One shows that the thermal states, the Hawking and the Unruh radiations, do not contribute significantly to the energy of the universe. Only the vacuum energy induced by the Hawking effect of hypothetical primordial black holes and the thermal vacuum of the intracluster medium (ICM), that is, the vacuum of hot plasma present at the center of a galaxy cluster, can represent a dark matter component. The vacuum of fields in curved space has a similar behavior [57].

A further discussion regarding the particle mixing phenomena, that is, the neutrino and quark mixing in the fermion sector and the axion-photon mixing and the meson mixing in the boson sector is required. For such systems, 
starting from the results of our previous works [44, 45, 49] and from the ones presented in $[56,58]$, obtained in a supersymmetric context, one shows that the flavor neutrino vacuum can give a contribution to the dark matter with a value compatible with its estimated upper bound, while the quark condensate, because of the quark confinement inside the hadrons, should not interact gravitationally. Moreover, one shows that the condensate of mixed boson $[42,43]$, as axions and axion-like particles (ALPs) in their interaction with photons, and of superpartners of mixed neutrinos can contribute to the dark energy with the state equation of the cosmological constant. It is expected that mixed particles like kaons, $B^{0}, D^{0}$ mesons, and $\eta-\eta^{\prime}$ system do not contribute to the energy on large scale, since they are unstable and not elementary particles.

We point out that the common origin of the nontrivial vacuum energy contributions of the systems described above resides in the fact that the physical vacuum of these systems is a condensate of couples of particles and antiparticles which lift the zero point energy to a positive value. The formal analogy (Bogoliubov transformations) among the disparate phenomena generating condensates could allow the simulation of the systems here analyzed by means of phenomena as the superconductivity, the Casimir effect, and the Schwinger effect, which are reproducible in table top experiments.

In the computations a particular care has been given to the renormalization procedure since different schemes may provide different renormalized expressions. The choice of the scheme to adopt is imposed by the need to preserve the symmetries of the system considered. For example, to preserve the Lorentz invariance of the Minkowski space-time, the dimensional regularization has been used in [59]. In this way, one has $p_{\text {vacuum }}=-\rho_{\text {vacuum }}$, which is the cosmological constant equation. On the contrary, in the case of curved spaces, as the Friedmann-Robertson-Walker background, the Lorentz invariance is no more a symmetry of the metric and a cut-off regularization can be utilized. In [57] a comoving cut-off on the momenta has been proposed. Notice that the cut-off regularization represents a valid choice also for the other vacuum condensates since the Lorentz invariance is not a symmetry of such systems. Motivated by such facts, in the following, the cut-off regularization will be used for all the phenomena considered, apart from the thermal vacuum for which the regularization is not needed.

The paper is structured as follows: in Section 2, the Bogoliubov transformations in QFT are introduced and the condensate structure of the transformed vacuum is shown. Section 3 presents the general form of the energy density and pressure of vacuum condensates for boson and fermion fields. Section 4 presents analysis of the contribution given to the energy of the universe by thermal states, with reference to the Hawking and Unruh effects. In Section 5 the fields in curved space are considered and in Section 6 the contributions to the dark energy and to the dark matter given by the particle mixing phenomena are presented. Section 7 is devoted to the conclusions.

\section{Bogoliubov Transformation and Vacuum Condensate}

The Bogoliubov transformations in the QFT context [60] describe disparate phenomena such as the Hawking-Unruh effect $[33,34]$, the Schwinger effect [35], the BCS theory of superconductivity [36], the Thermo Field Dynamics [38], the particle mixing phenomena [41-46], and the QFT in curved spacetimes [50]. For bosons and fermions they are given by

$$
\begin{gathered}
\tilde{a}_{\mathbf{k}}(\xi, t)=U_{\mathbf{k}}^{B} a_{\mathbf{k}}(t)-V_{-\mathbf{k}}^{B} a_{-\mathbf{k}}^{\dagger}(t), \\
\tilde{a}_{-\mathbf{k}}^{\dagger}(\xi, t)=U_{-\mathbf{k}}^{B *} a_{-\mathbf{k}}^{\dagger}(t)-V_{\mathbf{k}}^{B *} a_{\mathbf{k}}(t), \\
\widetilde{\alpha}_{\mathbf{k}}^{r}(\xi, t)=U_{\mathbf{k}}^{F} \alpha_{\mathbf{k}}^{r}(t)+V_{-\mathbf{k}}^{F} \alpha_{-\mathbf{k}}^{r \dagger}(t), \\
\widetilde{\alpha}_{-\mathbf{k}}^{r \dagger}(\xi, t)=U_{-\mathbf{k}}^{F *} \alpha_{-\mathbf{k}}^{r \dagger}(t)+V_{\mathbf{k}}^{F *} \alpha_{\mathbf{k}}^{r}(t),
\end{gathered}
$$

with $a_{\mathbf{k}}(t)=a_{\mathbf{k}} e^{-i \omega_{k} t}$ and $\alpha_{\mathbf{k}}^{r}(t)=\alpha_{\mathbf{k}}^{r} e^{-i \omega_{k} t}$, annihilators for bosons and fermion fields, respectively, such that $a_{\mathbf{k}}|0\rangle_{B}=$ $\alpha_{\mathbf{k}}^{r}|0\rangle_{F}=0$ and $\omega_{\mathbf{k}}=\sqrt{k^{2}+m^{2}}$. The coefficients satisfy the conditions $U_{\mathbf{k}}^{B}=U_{-\mathbf{k}}^{B}, V_{\mathbf{k}}^{B}=V_{-\mathbf{k}}^{B}$, and $\left|U_{\mathbf{k}}^{B}\right|^{2}-\left|V_{\mathbf{k}}^{B}\right|^{2}=1$ for bosons and $U_{\mathbf{k}}^{\psi}=U_{-\mathbf{k}}^{F}, V_{\mathbf{k}}^{F}=-V_{-\mathbf{k}}^{F}$, and $\left|U_{\mathbf{k}}^{F}\right|^{2}+\left|V_{\mathbf{k}}^{F}\right|^{2}=$ 1 , for fermions. Thus, they have the general form $U_{\mathbf{k}}^{B}=$ $e^{i \gamma_{1 \mathbf{k}}} \cosh \eta_{\mathbf{k}}(\zeta), V_{\mathbf{k}}^{B}=e^{i \gamma_{2 \mathbf{k}}} \sinh \eta_{\mathbf{k}}(\zeta), U_{\mathbf{k}}^{F}=e^{i \phi_{1 \mathbf{k}}} \cos \xi_{\mathbf{k}}(\zeta)$, and $V_{\mathbf{k}}^{F}=e^{i \phi_{2 \mathbf{k}}} \sin \xi_{\mathbf{k}}(\zeta)$, respectively. The parameter $\zeta$ controls the physics underlying the transformation. For example, $\zeta$ is related to the temperature $T$ in the Thermo Field Dynamics case or to the acceleration of the observer in the Unruh effect case. The phases $\phi_{i \mathbf{k}}$ and $\gamma_{i \mathbf{k}}$, with $i=1,2$, are irrelevant in our discussions.

Transformations (1) can be written at any time $t$ in terms of the generator $J_{\lambda}(\xi, t)$ as

$$
\chi_{\mathbf{k}}^{r}(\xi, t)=J_{\lambda}^{-1}(\xi, t) \chi_{\mathbf{k}}^{r}(t) J_{\lambda}(\xi, t),
$$

with $\chi=a, \alpha$ and $\lambda=B, F$. Similar relations hold for the creation operators. The generators have the property $J_{\lambda}^{-1}(\xi)=J_{\lambda}(-\xi)$. The vacua $|0(\xi, t)\rangle_{\lambda}$ annihilated by the new annihilators are related to the original ones $|0\rangle_{\lambda}$ by the formal relations $|0(\xi, t)\rangle_{\lambda}=J_{\lambda}^{-1}(\xi, t)|0\rangle_{\lambda}$, with $\lambda=F, B$.

This is a unitary operation if $\mathbf{k}$ assumes a discrete range of values, which happens in quantum mechanics in which there is a finite or countable number of canonical (anti)commutation relations CCRs. In this case, the Fock spaces built on the two vacua are equivalent and any vector in one space can be expressed in terms of a well defined sum of vectors in the other space. But in QFT, $\mathbf{k}$ assumes a continuous infinity of values; then one has, for bosons,

$$
|0(\xi, t)\rangle_{B}=\exp \left[-\delta(\mathbf{0}) \int d^{3} \mathbf{k} \log \cosh \xi_{\mathbf{k}}\right] \exp \left[\int d^{3} \mathbf{k} \tanh \xi_{\mathbf{k}}\left(a_{\mathbf{k}}^{\dagger}\right)^{2}\right]|0\rangle_{B}
$$


which is not a unitary transformation any more [61]. This shows that the vacuum $|0(\xi, t)\rangle_{B}$ cannot be expressed as a superposition of vectors in the Fock space built over $|0\rangle_{B}$. The same is true for the whole Fock space built over $|0(\xi, t)\rangle_{B}$; that is, the two Fock spaces are unitarily inequivalent [61]. Similar discussion holds for fermions.

In general, the existence in QFT of infinitely many representations which are unitarily inequivalent to each other, leads to the problem of the right choice of the Fock space and of the physical vacua associated with the particles which appear in observations. For the mentioned systems [33-50], ruled by Bogoliubov transformations, the physical vacua to be used in the computations are the $|0(\xi, t)\rangle_{\lambda}$ ones [61].

Notice that $|0(\xi, t)\rangle_{\lambda}$ is a condensate of couples of particles and antiparticles. Indeed one has

$$
{ }_{\lambda}\left\langle 0(\xi, t)\left|\chi_{\mathbf{k}}^{\dagger} \chi_{\mathbf{k}}\right| 0(\xi, t)\right\rangle_{\lambda}=\left|V_{\mathbf{k}}^{\lambda}\right|^{2},
$$

where $\chi=a, \alpha$ and $\lambda=B, F$, and (1) have been used. Such a condensate structure leads to an energy momentum tensor different from zero for $|0(\xi, t)\rangle_{\lambda}$.

\section{Energy Momentum Tensor of Vacuum Condensate}

One considers the free energy momentum tensor densities $T^{\mu \nu}(x)$ for real scalar fields $\phi, T_{B}^{\mu \nu}(x)=\partial_{\mu} \phi(x) \partial^{\mu} \phi(x)-$ $(1 / 2) g_{\mu \nu}\left(\partial^{\rho} \phi(x) \partial_{\rho} \phi(x)-m^{2} \phi(x)^{2}\right)$, and for Majorana fields $\psi, T_{F}^{\mu v}(x)=(i / 2) \bar{\psi}(x) \gamma_{\mu} \overleftrightarrow{\partial}^{\mu} \psi(x)$. One computes the expectation value of $T_{\mu \nu}^{\lambda}(x), \lambda=B, F$, on the transformed vacuum $|0(\xi, t)\rangle_{\lambda}$

$$
\begin{aligned}
\Xi_{\mu \nu}^{\lambda}(x) \equiv & { }_{\lambda}\left\langle 0(\xi, t)\left|: T_{\mu \nu}^{\lambda}(x):\right| 0(\xi, t)\right\rangle_{\lambda} \\
= & { }_{\lambda}\left\langle 0(\xi, t)\left|T_{\mu \nu}^{\alpha}(x)\right| 0(\xi, t)\right\rangle_{\lambda} \\
& { }_{-}\left\langle 0\left|T_{\mu \nu}^{\lambda}(x)\right| 0\right\rangle_{\lambda} .
\end{aligned}
$$

The symbol : $\cdots:$ denotes the normal ordering with respect to the original vacuum $|0\rangle_{\lambda}$. Notice that the off-diagonal components of the expectation value of $T_{\mu \nu}^{\lambda}(x)$ in (22) are zero, $\left\langle 0(\xi, t)\left|: T_{i, j}^{\lambda}(x):\right| 0(\xi, t)\right\rangle=0$, for $i \neq j$, being different from zero only the diagonal components. This implies that the condensates induced by Bogoliubov transformations behave as a perfect fluid and the energy density and pressure of boson and fermion condensates can be defined as

$$
\begin{aligned}
& \rho^{\lambda}=\left\langle 0(\xi, t)\left|: T_{00}^{\lambda}(x):\right| 0(\xi, t)\right\rangle, \\
& p^{\lambda}=\left\langle 0(\xi, t)\left|: T_{j j}^{\lambda}(x):\right| 0(\xi, t)\right\rangle,
\end{aligned}
$$

respectively.

In the boson case, one has

$$
\begin{aligned}
& \rho_{B}=\frac{1}{2}\left\langle 0(\xi, t)\left|:\left[\pi^{2}(x)+(\vec{\nabla} \phi(x))^{2}+m^{2} \phi^{2}(x)\right]:\right| 0(\xi, t)\right\rangle ; \\
& p_{B}=\left\langle 0(\xi, t)\left|:\left(\left[\partial_{j} \phi(x)\right]^{2}+\frac{1}{2}\left[\pi^{2}(x)-(\vec{\nabla} \phi(x))^{2}-m^{2} \phi^{2}(x)\right]\right):\right| 0(\xi, t)\right\rangle .
\end{aligned}
$$

In the particular case of the isotropy of the momenta, $k_{1}=k_{2}=k_{3}$, one has $\left[\partial_{j} \phi(x)\right]^{2}=(1 / 3)[\vec{\nabla} \phi(x)]^{2}$; then the pressure can be written as

$$
p_{B}=\frac{1}{2}\left\langle 0(\xi, t)\left|:\left[\pi^{2}(x)-\frac{1}{3}(\vec{\nabla} \phi(x))^{2}-m^{2} \phi^{2}(x)\right]:\right| 0(\xi, t)\right\rangle .
$$

In general, the vacuum condensates $|0(\xi, t)\rangle_{\lambda}$ are space or time dependent; therefore they violate the Lorentz invariance. This fact implies that the kinetic and gradient terms of (11) can be different from zero. Namely, $\left\langle 0(\xi, t)\left|: \pi^{2}(x):\right| 0(\xi, t)\right\rangle \neq 0$ and $\left\langle 0(\xi, t)\left|:[\vec{\nabla} \phi(x)]^{2}:\right| 0(\xi, t)\right\rangle \neq 0$. Therefore, from (8) and (11), one can have the following state equations: $w_{B}=p_{B} / \rho_{B}=$ 1 , if the kinetic term dominates; $w_{B}=-1 / 3$, if the gradient term dominates; and $w_{B}=-1$ (cosmological constant state equation) for dominating mass term. Moreover, the radiation state equation, $w_{B}=1 / 3$, can be achieved if the kinetic and gradient terms are of the same order, and the mass term is negligible. Such a result is achieved in the high momenta regime, $k \rightarrow \infty$. Finally, the state equation of the dark matter, $w_{B}=0$, is obtained for negligible gradient term and for kinetic and mass terms of the same order. This situation happens in the low momenta regime $k \rightarrow 0$.

If all the terms (kinetic, gradient, and mass ones) are taken into account, the energy density and pressure are given by 


$$
\begin{aligned}
& \rho_{B}=\int \frac{d^{3} \mathbf{k}}{(2 \pi)^{3}} \omega_{k}\left\langle 0(\xi, t)\left|a_{\mathbf{k}}^{\dagger} a_{\mathbf{k}}\right| 0(\xi, t)\right\rangle ; \\
& p_{B}=\int \frac{d^{3} \mathbf{k}}{(2 \pi)^{3}}\left[\frac{1}{3} \frac{k^{2}}{\omega_{k}}\left\langle 0(\xi, t)\left|a_{\mathbf{k}}^{\dagger} a_{\mathbf{k}}\right| 0(\xi, t)\right\rangle-\left(\frac{1}{3} \frac{k^{2}}{\omega_{k}}+\frac{1}{2} \frac{m^{2}}{\omega_{k}}\right)\left\langle 0(\xi, t)\left|\left(a_{\mathbf{k}} a_{-\mathbf{k}} e^{-i \omega_{k} t}+a_{\mathbf{k}}^{\dagger} a_{-\mathbf{k}}^{\dagger} e^{i \omega_{k} t}\right)\right| 0(\xi, t)\right\rangle\right],
\end{aligned}
$$

which explicitly become

$$
\begin{aligned}
\rho_{B} & =\frac{1}{2 \pi^{2}} \int_{0}^{\infty} d k k^{2} \omega_{k}\left|V_{k}^{B}\right|^{2}, \\
p_{B} & =\frac{1}{6 \pi^{2}} \int_{0}^{\infty} d k k^{2}\left[\frac{k^{2}}{\omega_{k}}\left|V_{k}^{B}\right|^{2}\right. \\
& \left.-\left(\frac{k^{2}}{\omega_{k}}+\frac{3 m^{2}}{2 \omega_{k}}\right)\left|U_{k}^{B}\right|\left|V_{k}^{B}\right| \cos \left(\omega_{k} t\right)\right] .
\end{aligned}
$$

The state equation is then

$$
\begin{aligned}
w_{B}= & \frac{1}{3} \frac{\int d^{3} \mathbf{k}\left(k^{2} / \omega_{k}\right)\left|V_{k}^{B}\right|^{2}}{\int d^{3} \mathbf{k} \omega_{k}\left|V_{k}^{B}\right|^{2}} \\
& -\frac{1}{3} \frac{\int d^{3} \mathbf{k}\left(k^{2} / \omega_{k}+3 m^{2} / 2 \omega_{k}\right) U_{k}^{B} V_{k}^{B} \cos \left(\omega_{k} t\right)}{\int d^{3} \mathbf{k} \omega_{k}\left|V_{k}^{B}\right|^{2}} .
\end{aligned}
$$
are

In the fermion case, the energy density and the pressure

$$
\begin{aligned}
& \rho_{F}=\frac{1}{2}\left\langle 0(\xi, t)\left|:\left[-i \bar{\psi} \gamma_{j} \partial^{j} \psi+m \bar{\psi} \psi\right]:\right| 0(\xi, t)\right\rangle \\
& p_{F}=\left\langle 0(\xi, t)\left|:\left(\frac{i}{2} \bar{\psi} \gamma_{j} \overleftrightarrow{\partial_{j}} \psi\right):\right| 0(\xi, t)\right\rangle
\end{aligned}
$$

In (16), one used the relation $(i / 2) \bar{\psi} \gamma_{0} \overleftrightarrow{\partial_{0}} \psi=i \bar{\psi} \gamma_{0} \partial_{0} \psi=$ $-i \bar{\psi} \gamma_{j} \partial^{j} \psi+m \bar{\psi} \psi$. By considering the following form of the energy momentum tensor density

$$
: T_{F}^{\mu \nu}:=:\left[\frac{i}{2} \bar{\psi} \gamma_{\mu} \overleftrightarrow{\partial}_{\nu} \psi-\eta_{\mu \nu}\left(\frac{i}{2} \bar{\psi} \gamma_{j} \overleftrightarrow{\partial}^{j} \psi-m \bar{\psi} \psi\right)\right]:
$$

one can obtain for fermion vacuum condensates the same state equations achieved for boson condensates (see above).
When all the terms are considered (kinematic, gradient, and mass ones), from (16) and (17), one has

$$
\begin{aligned}
& \rho_{F}=\sum_{r} \int \frac{d^{3} \mathbf{k}}{(2 \pi)^{3}}\left(\frac{k^{2}}{\omega_{k}}+\frac{m^{2}}{\omega_{k}}\right)\left\langle 0(\xi, t)\left|\alpha_{k}^{r \dagger} \alpha_{k}^{r}\right| 0(\xi, t)\right\rangle ; \\
& p_{F}=\frac{1}{3} \sum_{r} \frac{d^{3} \mathbf{k}}{(2 \pi)^{3}} \frac{k^{2}}{\omega_{k}}\left\langle 0(\xi, t)\left|\alpha_{k}^{r \dagger} \alpha_{k}^{r}\right| 0(\xi, t)\right\rangle,
\end{aligned}
$$

where $\alpha_{k}^{r}$ is the annihilator of fermion field, with $r=1,2$.

The explicit expressions of the energy density and pressure are

$$
\begin{aligned}
& \rho_{F}=\frac{1}{\pi^{2}} \int_{0}^{\infty} d k k^{2} \omega_{k}\left|V_{k}^{F}\right|^{2}, \\
& p_{F}=\frac{1}{3 \pi^{2}} \int_{0}^{\infty} d k \frac{k^{4}}{\omega_{k}}\left|V_{k}^{F}\right|^{2} .
\end{aligned}
$$

The state equation is then

$$
w_{F}=\frac{1}{3} \frac{\int d^{3} \mathbf{k}\left(k^{2} / \omega_{k}\right)\left|V_{k}^{F}\right|^{2}}{\int d^{3} \mathbf{k} \omega_{k}\left|V_{k}^{F}\right|^{2}} .
$$

The origin of the nonzero $\rho_{\lambda}$ and $p_{\lambda}$ is due to the fermionic and bosonic condensates structure of the physical vacuum which lifts the vacuum energy and pressure by positive amounts. Notice also that, being $J^{-1}(\xi, t)=J^{\dagger}(\xi, t)=$ $J(-\xi, t)$, one can write

$$
\begin{aligned}
{ }_{\lambda} & \left\langle 0(\xi, t)\left|: T_{\mu \nu}^{\lambda}(x):\right| 0(\xi, t)\right\rangle_{\lambda} \\
& ={ }_{\lambda}\left\langle 0\left|J_{\lambda}^{-1}(-\xi, t): T_{\mu \nu}^{\lambda}(x): J_{\lambda}(-\xi, t)\right| 0\right\rangle_{\lambda} .
\end{aligned}
$$

Such a property will be used in the following and the notation $\Theta(-\xi, x)=J^{-1}(-\xi, t) \Theta(x) J(-\xi, t)$ will be adopted to denote the operators transformed by the generator $J(-\xi, t)$.

Systems such as the thermal states and the particle mixing phenomenon have the generators $J$ 's which satisfy the condition $\vec{\nabla} J(\xi, t)=0$. Then, the energy density and pressure for bosons and fermions become

$$
\begin{aligned}
& \rho_{B}=\frac{1}{2}\left\langle 0\left|:\left[\pi^{2}(-\xi, x)+[\vec{\nabla} \phi(-\xi, x)]^{2}+m^{2} \phi^{2}(-\xi, x)\right]:\right| 0\right\rangle ; \\
& p_{B}=\left\langle 0\left|:\left(\left[\partial_{j} \phi(-\xi, x)\right]^{2}+\frac{1}{2}\left[\pi^{2}(-\xi, x)-[\vec{\nabla} \phi(-\xi, x)]^{2}-m^{2} \phi^{2}(-\xi, x)\right]\right):\right| 0\right\rangle, \\
& \rho_{F}=-i\left\langle 0\left|:\left[\psi^{\dagger}(-\xi, x) \gamma_{0} \gamma^{i} \partial_{i} \psi(-\xi, x)+m \psi^{\dagger}(-\xi, x) \gamma_{0} \psi(-\xi, x)\right]:\right| 0\right\rangle, \\
& p_{F}=i\left\langle 0\left|:\left[\psi^{\dagger}(-\xi, x) \gamma_{0} \gamma_{j} \partial_{j} \psi(-\xi, x)\right]:\right| 0\right\rangle,
\end{aligned}
$$


respectively. Equations (23)-(26) will be used to describe the vacuum contributions of the mixed particles. Notice that (23), (24) and (25), (26) do not coincide with the more general (8), (9) and (16), (17), respectively, since as a rule the operator $\nabla$ and the generators $J^{\prime}$ s do not commute.

Equations (8), (9) and (16), (17) hold for disparate physical phenomena. The explicit form of the Bogoliubov coefficients $U_{\mathbf{k}}^{\lambda}$ and $V_{\mathbf{k}}^{\lambda}, \lambda=B, F$, specifies the particular system. In the following only few phenomena will be considered in detail. However, the formal analogy among the systems characterized by the condensates, that is, the Bogoliubov transformations, permits extending the discussions contained in the next sections also to different phenomena, some of which are reproducible in laboratory.

\section{Thermal States, Hawking, and Unruh Effects}

We consider the formal framework of the Thermo Field Dynamics (TFD) [60-62], which has been successfully applied to a number of physical problems at nonzero temperature, in condensed matter physics, in nuclear physics, in particle physics, and in cosmology. In the case of systems at nonzero temperature, the physical vacuum is the thermal vacuum state $|0(\xi(\beta))\rangle_{\lambda}$, with $\lambda=B, F, \beta \equiv 1 /\left(k_{B} T\right)$, and $k_{B}$ being the Boltzmann constant [60-62]. The state $|0(\xi(\beta))\rangle_{\lambda}$ is defined in such a way that the thermal statistical average $\mathscr{N}_{\chi_{\mathbf{k}}}(\xi)$ is given by $\mathcal{N}_{\chi_{\mathbf{k}}}(\xi)={ }_{\lambda}\left\langle 0(\xi(\beta))\left|N_{\chi_{\mathbf{k}}}\right| 0(\xi(\beta))\right\rangle_{\lambda}$, with $N_{\chi_{\mathbf{k}}}=\chi_{\mathbf{k}}^{\dagger} \chi_{\mathbf{k}}(\chi=a, \alpha)$ being the number operator $[61,62]$. In the boson case, $|0(\xi(\beta))\rangle_{B}$ is expressed as

$$
|0(\xi(\beta))\rangle_{B}=\prod_{\mathbf{k}} \frac{1}{\cosh \xi_{k}} \exp \left(\tanh \xi_{k} a_{\mathbf{k}}^{\dagger} b_{\mathbf{k}}^{\dagger}\right)|0\rangle_{B},
$$

where $|0\rangle_{B}$ is the vacuum annihilated by $a_{\mathbf{k}}$ and $b_{\mathbf{k}}$. The auxiliary boson operator $b_{\mathbf{k}}$ commutes with $a_{\mathbf{k}}$ and is introduced in order to produce the trace operation in computing thermal averages. Similar discussions hold for fermions.

The thermal vacuum $|0(\xi(\beta))\rangle_{\lambda}$ is normalized to one, and in the infinite volume limit one has ${ }_{\lambda}\langle 0(\xi(\beta)) \quad \mid \quad 0\rangle_{\lambda} \rightarrow$ 0 as $V \rightarrow \infty, \forall \beta$. Moreover, for $\beta^{\prime} \neq \beta$, one has ${ }_{\lambda}\langle 0(\xi(\beta))|$ $\left.0\left(\xi\left(\beta^{\prime}\right)\right)\right\rangle_{\lambda} \rightarrow 0$ as $V \rightarrow \infty$. Therefore, $\left\{|0(\xi(\beta))\rangle_{\lambda}\right\}$ provides a representation of the CCR defined at each $\beta$ and unitarily inequivalent $\forall \beta^{\prime} \neq \beta$ to any other representation $\left\{\left|0\left(\beta^{\prime}\right)\right\rangle_{\lambda}\right\}$ in the infinite volume limit [60-62].

The annihilation operators of $|0(\xi(\beta))\rangle_{\lambda}$ (the tilde operators) are obtained by means of a Bogoliubov transformations similar to (1), with $a_{-\mathbf{k}}$ and $\tilde{a}_{-\mathbf{k}}(\xi, t)$ replaced for bosons by $b_{\mathbf{k}}$ and $\tilde{b}_{\mathbf{k}}(\xi(\beta))$, respectively, and $\alpha_{-\mathbf{k}}$ and $\tilde{\alpha}_{-\mathbf{k}}(\xi, t)$ replaced for fermions by the auxiliary operators $\beta_{\mathbf{k}}$ and $\widetilde{\beta}_{\mathbf{k}}(\xi(\beta))$, respectively. The Bogoliubov coefficients are given by $U_{\mathbf{k}}^{T}=$ $\sqrt{e^{\beta \omega_{\mathbf{k}}} /\left(e^{\beta \omega_{\mathbf{k}}} \pm 1\right)}$ and $V_{\mathbf{k}}^{T}=\sqrt{1 /\left(e^{\beta \omega_{\mathbf{k}}} \pm 1\right)}$, with - for bosons and + for fermions, where $\beta=1 / k_{B} T$ and $\omega_{\mathbf{k}}=\sqrt{k^{2}+m^{2}}$ [60-62]. Such coefficients, used in (13), (14), and (20) give the contributions of the thermal vacuum states to the energy and pressure. If one considers the cosmic microwave background temperature, that is, $T=2.7 \mathrm{~K}$, one has that the nonrelativistic particles give negligible contribution to the vacuum energy. Only photons and particles with masses of order of $\left(10^{-3}-10^{-4}\right) \mathrm{eV}$ can contribute significantly to the energy radiation [63]. Indeed in such cases one obtains energy densities of order of $10^{-51} \mathrm{GeV}^{4}$ and state equations, $w=1 / 3[63]$.

The thermal states can describe also the Unruh and the Hawking effects. The temperature is $T=\hbar a / 2 \pi c k_{b}$ for Unruh effect with $a$ being the acceleration of the observer and $T=\hbar c^{3} / 8 \pi G M k_{b}$ for the Hawking effect with $M$ being the black hole mass and $G$ gravitational constant. Both of the phenomena do not contribute to the energy of the universe since their temperature is very low. For example, a black hole of one solar mass has a temperature of only $60 \mathrm{nK}$ and the thermal vacuum energy of any particle is negligible. Only primordial black holes with very small mass could have temperatures higher than the one of the cosmic microwave background and then give a contribution to the energy of the universe.

A nontrivial contribution can be given by the thermal vacuum of the intracluster medium. Such hot plasma filling the center of galaxy clusters has temperatures of order of $(10 \div 100) \times 10^{6} \mathrm{~K}$. For example, the thermal vacuum of free electrons with temperature of $80 \times 10^{6} \mathrm{~K}$ has an energy of $10^{-47} \mathrm{GeV}^{4}$ and a state equation $w=0.01$. Such values are in agreement with the ones on the dark matter.

\section{Fields in Curved Background}

Another example of condensed vacuum system is represented by fields in curved spaces. In these cases, the energy momentum tensor for spin 0 and $1 / 2$ are given by [50]

$$
\begin{aligned}
T_{\mu \nu}^{s=0}(x)= & (1-2 \xi) \phi_{; \mu} \phi_{; \nu}+\left(2 \xi-\frac{1}{2}\right) g_{\mu \nu} g^{\rho \sigma} \phi_{; \rho} \phi_{; \sigma} \\
& -2 \xi \phi_{; \mu \nu} \phi+\frac{2}{n} \xi g_{\mu \nu} \phi \square \phi \\
& -\xi\left[R_{\mu \nu}-\frac{1}{2} R g_{\mu \nu}+\frac{2(n-1)}{n} \xi R g_{\mu \nu}\right] \phi^{2} \\
& +2\left[\frac{1}{4}-\left(1-\frac{1}{n}\right) \xi\right] m^{2} g_{\mu \nu} \phi^{2}, \\
& i \\
T_{\mu \nu}^{s=1 / 2}(x)= & \left.\frac{i}{2} \gamma_{(\mu} \nabla_{\nu)} \psi-\left(\nabla_{(\mu} \bar{\psi}\right) \gamma_{\nu)} \psi\right],
\end{aligned}
$$

respectively, and the energy density and pressure depend on the particular metric considered. Let us consider the spatially flat Friedmann-Robertson-Walker metric:

$$
d s^{2}=d t^{2}-a^{2}(t) d \mathbf{x}^{2}=a^{2}(\eta)\left(d \eta^{2}-d \mathbf{x}^{2}\right),
$$

where $a$ is the scale factor, $t$ is the comoving time, $\eta$ is the conformal time, and $\eta(t)=\int_{t_{0}}^{t}(d t / a(t))$, with $t_{0}$ being arbitrary constant. The boson field $\phi(\mathbf{x}, \eta)$ can be expressed as

$$
\phi(\mathbf{x}, \eta)=\int d^{3} \mathbf{k}\left[a_{\mathbf{k}} \phi_{k}(\eta)+a_{-\mathbf{k}}^{\dagger} \phi_{-k}^{*}(\eta)\right] e^{i \mathbf{k x}}
$$

where the mode functions $\phi_{k}(\eta)$ have analytical expression only in particular cases. However, in any case, the energy 
density and pressure, after the introduction of a cut-off on the momenta, can be written as $[64,65]$

$$
\begin{aligned}
& \rho_{\text {curv }}=\frac{2 \pi}{a^{2}} \int_{0}^{K} d k k^{2}\left(\left|\phi_{k}^{\prime}\right|^{2}+k^{2}\left|\phi_{k}\right|^{2}+m^{2}\left|\phi_{k}\right|^{2}\right), \\
& p_{\text {curv }}=\frac{2 \pi}{a^{2}} \int_{0}^{K} d k k^{2}\left(\left|\phi_{k}^{\prime}\right|^{2}-\frac{k^{2}}{3}\left|\phi_{k}\right|^{2}-m^{2}\left|\phi_{k}\right|^{2}\right) .
\end{aligned}
$$

In [57] it has been shown that at late time the cut-off on the momenta can be assumed to be much smaller than the comoving mass of the field, $K \ll m a$. Moreover, assuming that $m \gg H$, in an arbitrary Robertson-Walker metric for infrared regime, one obtains [57]

$$
\begin{aligned}
& \rho_{\text {curv }}=\frac{1}{8 \pi^{2}} \int_{0}^{K} d k k^{2}\left(\frac{2 m}{a^{3}}+\frac{9 H^{2}}{4 m a^{3}}+\frac{k^{2}}{m a^{5}}\right), \\
& p_{\text {curv }}=\frac{1}{8 \pi^{2}} \int_{0}^{K} d k k^{2}\left(\frac{9 H^{2}}{4 m a^{3}}-\frac{k^{2}}{3 m a^{5}}\right) .
\end{aligned}
$$

Therefore, state equation is $w_{\text {curv }} \simeq 0$; that is, the energy of the vacuum of a scalar field in curved space behaves as a dark matter component in the infrared regime. The energy density is [57]

$$
\rho_{\text {curv }}=\frac{m K^{3}}{12 \pi^{2} a^{3}} .
$$

The value of $\rho_{\text {curv }}$ depends on the values of the mass field $m$, on the scale factor $a$, and on the cut-off on the momenta $K$. Thus, numerical values compatible with the ones of dark matter can be found only for the values of the parameters such that $m K^{3} / a^{3} \sim 10^{-45} \mathrm{GeV}^{4}$.

We expect a similar result for fermion fields in curved spaces. Further study on such topics represents a work in progress.

\section{Particle Mixing}

The field mixing phenomenon is represented by the mixing of neutrinos and quarks in fermion sector and by the axionphoton mixing and the mixing of kaons, $B^{0}, D^{0}$, and $\eta-\eta^{\prime}$ systems in boson sector. For two fields, it is expressed, for both fermions and bosons, as

$$
\begin{aligned}
& \varphi_{1}(\theta, x)=\varphi_{1}(x) \cos (\theta)+\varphi_{2}(x) \sin (\theta), \\
& \varphi_{2}(\theta, x)=-\varphi_{1}(x) \sin (\theta)+\varphi_{2}(x) \cos (\theta),
\end{aligned}
$$

where $\theta$ is the mixing angle, $\varphi_{i}(\theta, x)$ are the mixed fields, and $\varphi_{i}(x)$ are the free fields, with $i=1,2$. In the case of neutrino mixing, the mixed fields are the flavor neutrino fields $\varphi_{1}(\theta, x) \equiv \nu_{e}$ and $\varphi_{2}(\theta, x) \equiv \nu_{\mu}$ and the free fields are the neutrinos with definite masses $m_{1}$ and $m_{2}, \varphi_{1}(x) \equiv \nu_{1}$ and $\varphi_{2}(x) \equiv \nu_{2}$. The two-flavor neutrino mixing case has been considered. In the boson sector, for axion-photon mixing in the presence of a magnetic field one has $\varphi_{1}(\theta, x) \equiv \gamma_{\|}(z)$ and $\varphi_{2}(\theta, x) \equiv a(z)$ (with $\gamma_{\|}(z)$ being photon polarization field parallel to the purely transverse magnetic field $\mathbf{B}=\mathbf{B}_{T}$ and $a$ axion field). The free fields are $\varphi_{1}(x) \equiv \gamma_{\|}^{\prime}(z)$ and $\varphi_{2}(x) \equiv a^{\prime}(z)$, with $\gamma_{\|}^{\prime}(z)=\gamma_{\|}^{\prime}(0) e^{-i \omega_{\gamma} z}, a^{\prime}(z)=a^{\prime}(0) e^{-i \omega_{a} z}$, and

$$
\begin{aligned}
& \omega_{\gamma}=\omega+\Delta_{-}, \\
& \omega_{a}=\omega+\Delta_{+}, \\
& \Delta_{ \pm}=-\frac{\omega_{P}^{2}+m_{a}^{2}}{4 \omega} \pm \frac{1}{4 \omega} \sqrt{\left(\omega_{P}^{2}-m_{a}^{2}\right)^{2}+\left(2 g \omega B_{T}\right)^{2}} .
\end{aligned}
$$

The mixing angle is $\theta_{a}=(1 / 2) \arctan \left(2 g \omega B_{T} /\left(m_{a}^{2}-\right.\right.$ $\left.\omega_{P}^{2}\right)$ ), where $g \in\left[10^{-16}-10^{-10}\right] \mathrm{GeV}^{-1}$ is the axion-photon coupling, $m_{a} \in\left[10^{-6}-10^{-2}\right] \mathrm{eV}$ is the axion mass, and $\omega_{P}$ is the plasma frequency. Moreover, for instable mesons, the mixed fields are $K^{0}$ and $\bar{K}^{0}, B^{0}$ and $\bar{B}^{0}$, or $D^{0}$ and $\bar{D}^{0}$. The corresponding free fields are $K_{L}$ and $K_{S}, B_{L}$ and $B_{H}$, and $D_{L}$ and $D_{H}$, respectively, and $\cos (\theta)=(1 / 2 p) \sqrt{1-z}$ and $\sin (\theta)=(1 / 2 p) \sqrt{1+z}$, with $q / p=\sqrt{\mathscr{H}_{21} / \mathscr{H}_{12}}\left(\mathscr{H}_{i j}\right.$ are the elements of the effective Hamiltonian $\mathscr{H}$ of mixed meson systems) and $z$ parameter describing the CPT violation.

The mixing transformations (35) can be written as $\varphi_{i}(\theta, x) \equiv J^{-1}(\theta, t) \varphi_{i}(x) J(\theta, t)$, where $i=1,2$ and $J(\theta, t)$ is the transformation generator $[41,42]$. Analogously, the mixed annihilation operators are $\chi_{\mathbf{k}, i}^{r}(\theta, t) \equiv J^{-1}(\theta, t) \chi_{\mathbf{k}, i}^{r}(t) J(\theta, t)$, with $\chi_{\mathbf{k}, i}^{r}=a_{\mathbf{k}, i}, \alpha_{\mathbf{k}, i}^{r}$ for bosons and fermion, respectively, and $i=1,2$. They annihilate the mixed vacuum $|0(\theta, t)\rangle \equiv$ $J^{-1}(\theta, t)|0\rangle_{1,2}$, where $|0\rangle_{1,2}$ is the vacuum annihilated by $\chi_{\mathbf{k}, i}^{r}$.

The physical vacuum where particle oscillations appear is $|0(\theta, t)\rangle$. It is a (coherent) condensate of $\chi_{\mathbf{k}, i}$ particles (antiparticles) [41, 42]:

$$
\left\langle 0(\theta, t)\left|\chi_{\mathbf{k}, i}^{r \dagger} \chi_{\mathbf{k}, i}^{r}\right| 0(\theta, t)\right\rangle=\sin ^{2} \theta\left|\Upsilon_{\mathbf{k}}^{\lambda}\right|^{2}
$$

where $\lambda=B, F$ and $i=1,2$ and the reference frame $\mathbf{k}=(0,0,|\mathbf{k}|)$ has been adopted for convenience. $\Upsilon_{\mathbf{k}}^{\lambda}$ is the Bogoliubov coefficient entering the mixing transformation. For boson and fermion one has $[41,42]$

$$
\begin{aligned}
& \left|Y_{\mathbf{k}}^{B}\right|=\frac{1}{2}\left(\sqrt{\frac{\Omega_{k, 1}}{\Omega_{k, 2}}}-\sqrt{\frac{\Omega_{k, 2}}{\Omega_{k, 1}}}\right), \\
& \left|Y_{\mathbf{k}}^{F}\right|=\frac{\left(\Omega_{k, 1}+m_{1}\right)-\left(\Omega_{k, 2}+m_{2}\right)}{2 \sqrt{\Omega_{k, 1} \Omega_{k, 2}\left(\Omega_{k, 1}+m_{1}\right)\left(\Omega_{k, 2}+m_{2}\right)}}|\mathbf{k}|,
\end{aligned}
$$

respectively, with $\left|\Sigma_{\mathbf{k}}^{B}\right|^{2}-\left|Y_{\mathbf{k}}^{B}\right|^{2}=1$ and $\left|\Sigma_{\mathbf{k}}^{F}\right|^{2}+\left|\Upsilon_{\mathbf{k}}^{F}\right|^{2}=1\left(\Sigma_{\mathbf{k}}^{\lambda}\right.$ are the other coefficients entering in the transformations) and $\Omega_{k, i}$ are energies of the free fields; for example, $\Omega_{k, i}=\omega_{\gamma}, \omega_{a}$ for axions-photon mixing and $\Omega_{k, i}=\omega_{k, i}$ for neutrinos, $i=$ 1,2 . 
6.1. Boson Mixing. For mixed bosons, (23) and (24) become

$$
\begin{aligned}
\rho_{\text {mix }}^{B} & =\left\langle 0(\theta, t)\left|: T_{00}^{B-\text { mix }}(x):\right| 0(\theta, t)\right\rangle=\frac{1}{2}\left\langle 0\left|: \sum_{i}\left[\pi_{i}^{2}(-\theta, x)+\left[\vec{\nabla} \phi_{i}(-\theta, x)\right]^{2}+m_{i}^{2} \phi_{i}^{2}(-\theta, x)\right]:\right| 0\right\rangle \\
p_{\text {mix }}^{B} & =\left\langle 0(\theta, t)\left|: T_{j j}^{B-\text { mix }}(x):\right| 0(\theta, x)\right\rangle \\
& =\left\langle 0\left|: \sum_{i}\left(\left[\partial_{j} \phi_{i}(-\theta, x)\right]^{2}+\frac{1}{2}\left[\pi_{i}^{2}(-\theta, x)-\left[\vec{\nabla} \phi_{i}(-\theta, x)\right]^{2}-m_{i}^{2} \phi_{i}^{2}(-\theta, x)\right]\right):\right| 0\right\rangle,
\end{aligned}
$$

respectively, where

$$
\begin{aligned}
& \pi_{1}(-\theta, x)=\pi_{1}(x) \cos (\theta)-\pi_{2}(x) \sin (\theta), \\
& \pi_{2}(-\theta, x)=\pi_{1}(x) \sin (\theta)+\pi_{2}(x) \cos (\theta) .
\end{aligned}
$$

From (42), one can immediately see that $\sum_{i} \pi_{i}^{2}(-\theta, x)=$ $\sum_{i} \pi_{i}^{2}(x), \sum_{i}\left[\vec{\nabla} \phi_{i}(-\theta, x)\right]^{2}=\sum_{i}\left[\vec{\nabla} \phi_{i}(x)\right]^{2}$, and $\sum_{i}\left[\partial_{j} \phi_{i}(-\theta, x)\right]^{2}=$ $\sum_{i}\left[\partial_{j} \phi_{i}(x)\right]^{2}$; that is, such operators are invariant under the action of the generator $J(-\theta, t)$. This fact implies that the kinetic and gradient terms of the mixed vacuum are equal to zero

$$
\begin{aligned}
\left\langle 0\left|: \sum_{i} \pi_{i}^{2}(-\theta, x):\right| 0\right\rangle=\left\langle 0\left|: \sum_{i}\left[\vec{\nabla} \phi_{i}(-\theta, x)\right]^{2}:\right| 0\right\rangle \\
=\left\langle 0\left|: \sum_{i}\left[\partial_{j} \phi_{i}(-\theta, x)\right]^{2}:\right| 0\right\rangle=0 .
\end{aligned}
$$

Then, (40) and (41) become

$$
\begin{aligned}
& \rho_{\text {mix }}^{B}=\left\langle 0\left|: \sum_{i} m_{i}^{2} \phi_{i}^{2}(-\theta, x):\right| 0\right\rangle, \\
& p_{\text {mix }}^{B}=-\left\langle 0\left|: \sum_{i} m_{i}^{2} \phi_{i}^{2}(-\theta, x):\right| 0\right\rangle,
\end{aligned}
$$

and the state equation is $w_{\text {mix }}^{B}=-1$ (which is the state equation of the cosmological constant) independent of the choice of the cut-off on the momenta. Similar result has been obtained in supersymmetric context in $[56,58]$. Here one analyzes the possible phenomenological implications of such result. Denoting $\Delta m^{2}=\left|m_{2}^{2}-m_{1}^{2}\right|$, the energy density of the boson mixed vacuum is explicitly given by

$$
\rho_{\text {mix }}^{B}=\frac{\Delta m^{2} \sin ^{2} \theta}{8 \pi^{2}} \int_{0}^{K} d k k^{2}\left(\frac{1}{\omega_{k, 1}}-\frac{1}{\omega_{k, 2}}\right),
$$

where $K$ is the cut-off on the momenta. Equation (46) will be now solved for the mixing of axion-like particles and for the flavor mixing of supersymmetric partners of neutrinos.

Contribution of Axion-Like Particles. In the case of the mixing between the photon and the axion-like particles, denoting with $m_{1}=m_{\gamma}=0$, the photon mass and with $m_{2}=m_{a}$, the axion mass, one has

$$
\begin{gathered}
\rho_{\text {mix }}^{\text {axion }}=\frac{m_{a}^{2} \sin ^{2} \theta_{a}}{16 \pi^{2}}\left[K\left(K-\sqrt{K^{2}+m_{a}^{2}}\right)\right. \\
\left.+m_{a}^{2} \log \left(\frac{K+\sqrt{K^{2}+m_{a}^{2}}}{m_{a}}\right)\right] .
\end{gathered}
$$

In astrophysical contexts, as in the case of active galactic nuclei, quasars, supernova, and magnetars, the magnetic field strength varies as $B \in\left[10^{6}-10^{17}\right] G$. Also the plasma frequencies and the photon energies $\omega$ vary considerably. Therefore, the mixing angles $\theta_{a}$ depend on the particular system one considers.

Notice, however, that for axion mass $m_{a}$ of order of $2 \times$ $10^{-2} \mathrm{eV}$ and $\sin _{a}^{2} \theta \sim 10^{-2}$ (which could be obtained for different astrophysical objects), using the Planck scale cutoff, $K \sim 10^{19} \mathrm{GeV}$, one has $\rho_{\text {mix }}^{\text {axion }}=2.3 \times 10^{-47} \mathrm{GeV}^{4}$, which is compatible with the estimated upper bound on the dark energy.

Smaller values of $m_{a}$ or of $\sin ^{2} \theta \sim 10^{-2}$ lead to smaller values of $\rho_{\text {mix }}^{\text {axion }}$.

Contribution of Neutrino Superpartners. In the case superpartners of the neutrinos, integral (46) leads to

$$
\begin{aligned}
\rho_{\text {mix }}^{B} & =\frac{\Delta m^{2} \sin ^{2} \theta}{16 \pi^{2}}\left[K\left(\sqrt{K^{2}+m_{1}^{2}}-\sqrt{K^{2}+m_{2}^{2}}\right)\right. \\
& +m_{2}^{2} \log \left(\frac{K+\sqrt{K^{2}+m_{2}^{2}}}{m_{2}}\right) \\
& \left.-m_{1}^{2} \log \left(\frac{K+\sqrt{K^{2}+m_{1}^{2}}}{m_{1}}\right)\right] .
\end{aligned}
$$

One considers then masses similar to the ones of the neutrinos, $m_{1}=10^{-3} \mathrm{eV}$ and $m_{2}=9 \times 10^{-3} \mathrm{eV}$, in order that $\Delta m^{2}=8 \times 10^{-5} \mathrm{eV}^{2}$. Moreover, one assumes $\sin ^{2} \theta=0.3$. One obtains $\rho_{\text {mix }}^{B}=7 \times 10^{-47} \mathrm{GeV}^{4}$ for a cut-off on the momenta 
$K=10 \mathrm{eV}$ and $\rho_{\text {mix }}^{B}=6.9 \times 10^{-46} \mathrm{GeV}^{4}$ for a cut-off of order of the Planck scale $10^{19} \mathrm{GeV}$. Smaller values of the mixing angle lead to values which are compatible with the estimated value of the dark energy also in the case in which the cut-off is $K=10^{19} \mathrm{GeV}$; indeed $\rho_{\text {mix }}^{B}$ depends linearly on $\sin ^{2} \theta$.

6.2. Fermion Mixing. In the case of fermion mixing, (25) and (26) become

$$
\begin{aligned}
& \rho_{\text {mix }}^{F}=-\langle 0|: \sum_{i}\left[\psi_{i}^{\dagger}(-\theta, x) \gamma_{0} \gamma^{j} \partial_{j} \psi_{i}(-\theta, x)\right. \\
& \left.\quad+m \psi_{i}^{\dagger}(-\theta, x) \gamma_{0} \psi_{i}(-\theta, x)\right]:|0\rangle ; \\
& p_{\text {mix }}^{F}=i\left\langle 0\left|: \sum_{i}\left[\psi_{i}^{\dagger}(-\theta, x) \gamma_{0} \gamma_{j} \partial_{j} \psi_{i}(-\theta, x)\right]:\right| 0\right\rangle,
\end{aligned}
$$

where $\psi_{i}(-\theta, x)$ are the flavor neutrino fields or the quark fields. By

$$
\begin{gathered}
\sum_{i} \bar{\psi}_{i}(-\theta, x) \gamma^{j} \partial_{j} \psi_{i}(-\theta, x)=\sum_{i} \bar{\psi}_{i}(x) \gamma^{j} \partial_{j} \psi_{i}(x) \\
\sum_{i}\left[\psi_{i}^{\dagger}(-\theta, x) \gamma_{0} \gamma_{j} \partial_{j} \psi_{i}(-\theta, x)\right] \\
\quad=\sum_{i}\left[\psi_{i}^{\dagger}(x) \gamma_{0} \gamma_{j} \partial_{j} \psi_{i}(x)\right]
\end{gathered}
$$

then

$$
\begin{aligned}
\langle 0| & : \sum_{i} \bar{\psi}_{i}(-\theta, x) \gamma^{j} \partial_{j} \psi_{i}(-\theta, x):|0\rangle \\
& =\left\langle 0\left|: \sum_{i}\left[\psi_{i}^{\dagger}(-\theta, x) \gamma_{0} \gamma_{j} \partial_{j} \psi_{i}(-\theta, x)\right]:\right| 0\right\rangle=0 .
\end{aligned}
$$

Thus, the energy density and pressures of (49) and (50) become

$$
\begin{aligned}
& \rho_{\text {mix }}^{F}=-\left\langle 0\left|: \sum_{i}\left[m_{i} \psi_{i}^{\dagger}(-\theta, x) \gamma_{0} \psi_{i}(-\theta, x)\right]:\right| 0\right\rangle, \\
& p_{\text {mix }}^{F}=0
\end{aligned}
$$

respectively. The pressure of the fermion mixed vacuum $|0(\theta, t)\rangle$ is equal to zero independent of the regularization adopted. Then the state equation in this case is $w_{\text {mix }}^{F}=0$, which is the one of the dark matter. Similar result has been obtained in supersymmetric context in $[56,58]$. Now one shows the consequences of such a result. Solving (53), the energy density of the fermion mixed vacuum is

$$
\rho_{\text {mix }}^{F}=\frac{\Delta m \sin ^{2} \theta}{2 \pi^{2}} \int_{0}^{K} d k k^{2}\left(\frac{m_{2}}{\omega_{k, 2}}-\frac{m_{1}}{\omega_{k, 1}}\right),
$$

and explicitly one has

$$
\begin{aligned}
\rho_{\text {mix }}^{F} & =\frac{\Delta m \sin ^{2} \theta}{2 \pi^{2}}\left[K\left(m_{2} \sqrt{K^{2}+m_{2}^{2}}-m_{1} \sqrt{K^{2}+m_{1}^{2}}\right)\right. \\
& -m_{2}^{3} \log \left(\frac{K+\sqrt{K^{2}+m_{2}^{2}}}{m_{2}}\right) \\
& \left.+m_{1}^{3} \log \left(\frac{K+\sqrt{K^{2}+m_{1}^{2}}}{m_{1}}\right)\right] .
\end{aligned}
$$

For masses of order of $10^{-3} \mathrm{eV}$, such that $\Delta m^{2}$ is of order of $8 \times 10^{-5} \mathrm{eV}^{2}$ and a cut-off on the momenta $K=m_{1}+m_{2}$, one obtains $\rho_{\text {mix }}^{F}=4 \times 10^{-47} \mathrm{GeV}^{4}$, which is in agreement with the estimated upper bound of the dark matter. A possible mechanism which imposes a very low cut-off for neutrinos is given in [66]. Values of $K$ of order of the Plank scale lead to $\rho_{\text {mix }}^{F} \sim \times 10^{-46} \mathrm{GeV}^{4}$.

We point out that we have considered an empty universe without matter and gravitational interaction. This fact produces the homogeneity of the condensates. The presence of matter interacting with the neutrino flavor vacuum and with the vacuum condensates presented above could generate clustered matter and therefore the irregularities which are observed in the universe.

By considering the quark masses, the value of $\rho_{\text {mix }}^{F}$ one obtains is much higher than the ones obtained above. However, the quark confinement inside the hadrons should inhibit the gravitational interaction of the quark vacuum condensate. Therefore such condensate should play no role in the formation of structures on large scale and it should not affect the dark matter component.

Moreover, the condensates induced by the mixed bosons, which behave as a cosmological constant, can evolve remaining homogeneous also in the presence of matter, since their pressure is negative.

\section{Conclusions}

It has been shown that the vacuum condensates induced by different phenomena can contribute to the dark sector of the universe. The vacuum states of these systems are indeed condensates of couples of particles and antiparticles which generate nonzero vacuum energies and which, under particular conditions, behave as dark matter or dark energy.

The contributions given by the thermal states, by the fields in curved space, and by the particle mixing phenomenon have been analyzed. It has been shown that the thermal states and the condensates due to Unruh and Hawking effects do not contribute considerably to the vacuum energy. Nontrivial contributions to the energy are given by the thermal vacuum of the intercluster medium, by the vacuum of fields in curved space-time, and by the flavor vacuum of neutrinos. Such vacuum condensates have a negligible pressure; therefore, their state equations are similar to the dark matter one. 
Moreover the values of the energies are compatible with the one estimated for the dark matter.

On the other hand, the mixing between photons and axion-like particles can reproduce the behavior and the estimated value of the dark energy component.

The formal analogy existing among completely different phenomena, characterized by vacuum condensates, suggests investigating the properties of the condensates of the systems here studied, in phenomena like the superconductivity, the Casimir effect, and the Schwinger effect, which can be analyzed in table top experiments. Therefore, the results presented in this paper can open a completely new way in the research of dark matter and in the study of the dark energy.

\section{Competing Interests}

The author declares that there is no conflict of interests regarding the publication of this paper.

\section{Acknowledgments}

Partial financial support from MIUR is acknowledged.

\section{References}

[1] P. de Bernardis, P. A. R. Ade, J. J. Bock et al., "A flat Universe from high-resolution maps of the cosmic microwave background radiation," Nature, vol. 404, pp. 955-959, 2000.

[2] D. N. Spergel, L. Verde, H. V. Peiris et al., "First-year Wilkinson Microwave Anisotropy Probe (WMAP) ${ }^{*}$ observations: determination of cosmological parameters," The Astrophysical Journal Supplement Series, vol. 148, no. 1, pp. 175-194, 2003.

[3] S. Dodelson, V. K. Narayanan, M. Tegmark et al., "The threedimensional power spectrum from angular clustering of galaxies in early sloan digital sky survey data," The Astrophysical Journal, vol. 572, no. 1, p. 140, 2002.

[4] A. S. Szalay, B. Jain, T. Matsubara et al., "Karhunen-Loève estimation of the power spectrum parameters from the angular distribution of galaxies in early sloan digital sky survey data," The Astrophysical Journal, vol. 591, pp. 1-11, 2003.

[5] A. G. Riess, L.-G. Strolger, J. Tonry et al., "Type Ia Supernova Discoveries at $z>1$ from the hubble space telescope: evidence for past deceleration and constraints on dark energy evolution," The Astrophysical Journal, vol. 607, no. 2, pp. 665-687, 2004.

[6] L. Amendola and S. Tsujikawa, Dark Energy: Theory and Observations, Cambridge University Press, New York, NY, USA, 2010.

[7] S. Nojiri and S. D. Odintsov, "Unified cosmic history in modified gravity: from $\mathrm{F}(\mathrm{R})$ theory to Lorentz non-invariant models," Physics Reports, vol. 505, no. 2-4, pp. 59-144, 2011.

[8] A. Silvestri and M. Trodden, "Approaches to understanding cosmic acceleration," Reports on Progress in Physics, vol. 72, no. 9, Article ID 096901, 28 pages, 2009.

[9] J. A. Frieman, M. S. Turner, and D. Huterer, "Dark energy and the accelerating universe," Annual Review of Astronomy and Astrophysics, vol. 46, pp. 385-432, 2008.

[10] R. Durrer and R. Maartens, "Dark energy and dark gravity: theory overview," General Relativity and Gravitation, vol. 40, no. 2, pp. 301-328, 2008.
[11] S. Capozziello and G. Lambiase, "A comprehensive view of cosmological Dark Side," https://arxiv.org/abs/1304.5640.

[12] M. Sami, "Models of dark energy," in The Invisible Universe: Dark Matter and Dark Energy, vol. 720 of Lecture Notes in Physics, pp. 219-256, Springer, Berlin, Germany, 2007.

[13] E. J. Copeland, M. Sami, and S. Tsujikawa, "Dynamics of dark energy," International Journal of Modern Physics D, vol. 15, no. 11, pp. 1753-1935, 2006.

[14] S. Capozziello, "Curvature quintessence," International Journal of Modern Physics D, vol. 11, no. 4, pp. 483-491, 2002.

[15] S. Capozziello and G. Lambiase, "Higher-order corrections to the effective gravitational action from Noether symmetry approach," General Relativity and Gravitation, vol. 32, no. 2, pp. 295-311, 2000.

[16] G. Lambiase, S. Mohanty, and A. R. Prasanna, "Neutrino coupling to cosmological background: a review on gravitational baryo/leptogenesis," International Journal of Modern Physics D, vol. 22, no. 12, Article ID 1330030, 2013.

[17] T. P. Sotiriou and V. Faraoni, " $f(R)$ theories of gravity," Reviews of Modern Physics, vol. 82, p. 451, 2010.

[18] A. De Felice and S. Tsujikawa, " $\mathrm{f}(\mathrm{R})$ theories," Living Reviews in Relativity, vol. 13, p. 3, 2010.

[19] R. Schutzhold, "Josephson junction through a thin ferromagnetic layer: negative coupling," Physical Review Letters, vol. 89, no. 13, Article ID 081302, 2002.

[20] E. C. Thomas, F. R. Urban, and A. R. Zhitnitsky, "The cosmological constant as a manifestation of the conformal anomaly?" Journal of High Energy Physics, vol. 2009, no. 8, article 043, 2009.

[21] F. R. Klinkhamer and G. E. Volovik, "Gluonic vacuum, $q$-theory, and the cosmological constant," Physical Review D, vol. 79, Article ID 063527, 2009.

[22] F. R. Urban and A. R. Zhitnitsky, "Cosmological constant from the ghost: a toy model," Physical Review D, vol. 80, no. 6, Article ID 063001, 2009.

[23] F. R. Klinkhamer and G. E. Volovik, "Vacuum energy density kicked by the electroweak crossover," Physical Review D, vol. 80, no. 8, Article ID 083001, 2009.

[24] S. Alexander, T. Biswas, and G. Calcagni, "Cosmological Bardeen-Cooper-Schrieffer condensate as dark energy," Physical Review D, vol. 81, no. 4, Article ID 043511, 13 pages, 2010.

[25] N. J. Poplawski, "Cosmological constant from quarks and torsion," Annalen der Physik, vol. 523, pp. 291-295, 2011.

[26] G. Bertone, D. Hooper, and J. Silk, "Particle dark matter: evidence, candidates and constraints," Physics Reports, vol. 405, no. 5-6, pp. 279-390, 2005.

[27] G. D’Amico, M. Kamionkowski, and K. Sigurdson, "Dark matter astrophysics," https://arxiv.org/abs/0907.1912.

[28] G. Bertone, Ed., Particle Dark Matter: Observations, Models and Searches, Institut d'Astrophysique, Paris, France, Cambridge University Press, Cambridge, UK, 2010.

[29] A. Bottino and N. Fornengo, "Dark matter and its particle candidates," Trieste 1998, Non-Accelerator Particle Physics, 1988.

[30] S. Ferraro, F. Schmidt, and W. Hu, "Cluster abundance in $f(R)$ gravity models," Physical Review D, vol. 83, no. 6, Article ID 063503, 8 pages, 2011.

[31] L. Lombriser, F. Schmidt, T. Baldauf, R. Mandelbaum, U. Seljak, and R. E. Smith, "Cluster density profiles as a test of modified gravity," Physical Review D, vol. 85, no. 10, Article ID 102001, 2012. 
[32] F. Schmidt, A. Vikhlinin, and W. Hu, "Cluster constraints on $f(R)$ gravity," Physical Review D, vol. 80, no. 8, Article ID 083505, 2009.

[33] S. W. Hawking, "Particle creation by black holes," Communications in Mathematical Physics, vol. 46, no. 3, pp. 199-220, 1975, [Erratum-ibid., vol. 46, pp. 199, 1976].

[34] W. G. Unruh, "Notes on black-hole evaporation," Physical Review D, vol. 14, no. 4, p. 870, 1976.

[35] J. S. Schwinger, "On gauge invariance and vacuum polarization," Physical Review, vol. 82, no. 5, pp. 664-679, 1951.

[36] J. Bardeen, L. N. Cooper, and J. R. Schrieffer, "Theory of superconductivity," Physical Review, vol. 108, no. 5, pp. 11751204, 1957.

[37] A. Iorio, "Weyl-gauge symmetry of graphene," Annals of Physics, vol. 326, no. 5, pp. 1334-1353, 2011.

[38] Y. Takahashi and H. Umezawa, "Thermo field dynamics," Collective Phenomena, vol. 2, pp. 55-80, 1975.

[39] H. B. G. Casimir and D. Polder, "The influence of retardation on the London-van der Waals forces," Physical Review, vol. 73, no. 4, pp. 360-372, 1948.

[40] H. B. G. Casimir, "On the attraction between two perfectly conducting plates," Proceedings of the Koninklijke Nederlandse Akademie van Wetenschappen, vol. 51, pp. 793-795, 1948.

[41] M. Blasone, A. Capolupo, and G. Vitiello, "Quantum field theory of three flavor neutrino mixing and oscillations with CP violation," Physical Review D, vol. 66, no. 2, Article ID 025033, 2002.

[42] M. Blasone, A. Capolupo, O. Romei, and G. Vitiello, "Quantum field theory of boson mixing," Physical Review D, vol. 63, no. 12, Article ID 125015, 9 pages, 2001.

[43] A. Capolupo, C.-R. Ji, Y. Mishchenko, and G. Vitiello, "Phenomenology of flavor oscillations with non-perturbative effects from quantum field theory," Physics Letters B, vol. 594, no. 1-2, pp. 135-140, 2004.

[44] A. Capolupo, S. Capozziello, and G. Vitiello, "Neutrino mixing as a source of dark energy," Physics Letters A, vol. 363, no. 1-2, pp. 53-56, 2007.

[45] A. Capolupo, S. Capozziello, and G. Vitiello, "Dark energy and particle mixing," Physics Letters A, vol. 373, no. 6, pp. 601-610, 2009.

[46] A. Capolupo, S. Capozziello, and G. Vitiello, "Dark energy, cosmological constant and neutrino mixing," International Journal of Modern Physics A, vol. 23, no. 31, pp. 4979-4990, 2008.

[47] M. Blasone, A. Capolupo, S. Capozziello, and G. Vitiello, "Neutrino mixing, flavor states and dark energy," Nuclear Instruments and Methods in Physics Research A, vol. 588, no. 1-2, pp. 272-275, 2008.

[48] M. Blasone, A. Capolupo, and G. Vitiello, "Particle mixing, flavor condensate and dark energy," Progress in Particle and Nuclear Physics, vol. 64, no. 2, pp. 451-453, 2010.

[49] M. Blasone, A. Capolupo, S. Capozziello, S. Carloni, and G. Vitiello, "Neutrino mixing contribution to the cosmological constant," Physics Letters A, vol. 323, no. 3-4, pp. 182-189, 2004.

[50] N. D. Birrell and P. C. W. Davies, Quantum Fields in Curved Space, Cambridge University Press, Cambridge, UK, 1984.

[51] A. Capolupo, M. Di Mauro, and A. Iorio, "Mixing-induced spontaneous supersymmetry breaking," Physics Letters A, vol. 375, no. 39, pp. 3415-3418, 2011.
[52] A. Capolupo and M. Di Mauro, "Spontaneous supersymmetry breaking induced by vacuum condensates," Physics Letters A, vol. 376, no. 45, pp. 2830-2833, 2012.

[53] A. Capolupo and M. Di Mauro, "Vacuum condensates, flavor mixing and spontaneous supersymmetry breaking," Acta Physica Polonica B, vol. 44, no. 1, pp. 81-89, 2013.

[54] A. Capolupo and G. Vitiello, "Spontaneous supersymmetry breaking probed by geometric invariants," Advances in High Energy Physics, vol. 2013, Article ID 850395, 5 pages, 2013.

[55] A. Capolupo and M. Di Mauro, "Vacuum condensates as a mechanism of spontaneous supersymmetry breaking," Advances in High Energy Physics, vol. 2015, Article ID 929362, 6 pages, 2015.

[56] N. E. Mavromatos, S. Sarkar, and W. Tarantino, "D-foaminduced flavor condensates and breaking of supersymmetry in free Wess-Zumino fluids," Physical Review D, vol. 84, Article ID 044050, 2011.

[57] F. D. Albareti, J. A. R. Cembranos, and A. L. Maroto, "Vacuum energy as dark matter," Physical Review D, vol. 90, no. 12, Article ID 123509, 2014.

[58] W. Tarantino, "Dark matter and dark energy via nonperturbative (flavor) vacua," Physical Review D, vol. 85, no. 4, Article ID 045020, 2012.

[59] E. K. Akhmedov, "Vacuum energy and relativistic invariance," https://arxiv.org/abs/hep-th/0204048.

[60] H. Umezawa, Advanced Field Theory: Micro, Macro, and Thermal Physics, AIP, New York, NY, USA, 1993.

[61] H. Umezawa, H. Matsumoto, and M. Tachiki, Thermo Field Dynamics and Condensed States, North-Holland Publishing Company, 1982.

[62] Y. Takahashi and H. Umezawa, "Thermo field dynamics," Collect. Phenom., vol. 2, p. 55, 1975, Reprinted in: International Journal of Modern Physics B, vol. 10, p. 1755, 1996.

[63] A. Capolupo, G. Lambiase, and G. Vitiello, "Thermal condensate structure and cosmological energy density of the universe," Advances in High Energy Physics, vol. 2016, Article ID 3127597, 6 pages, 2016.

[64] L. Parker and S. A. Fulling, "Adiabatic regularization of the energy-momentum tensor of a quantized field in homogeneous spaces," Physical Review D, vol. 9, no. 2, pp. 341-354, 1974.

[65] S. A. Fulling and L. Parker, "Renormalization in the theory of a quantized scalar field interacting with a Robertson-Walker space-time," Annalen der Physik D, vol. 87, pp. 176-204, 1974.

[66] N. E. Mavromatos and S. Sarkar, "Towards a microscopic construction of flavour vacua from a space-time foam model," New Journal of Physics, vol. 10, Article ID 073009, 2008. 

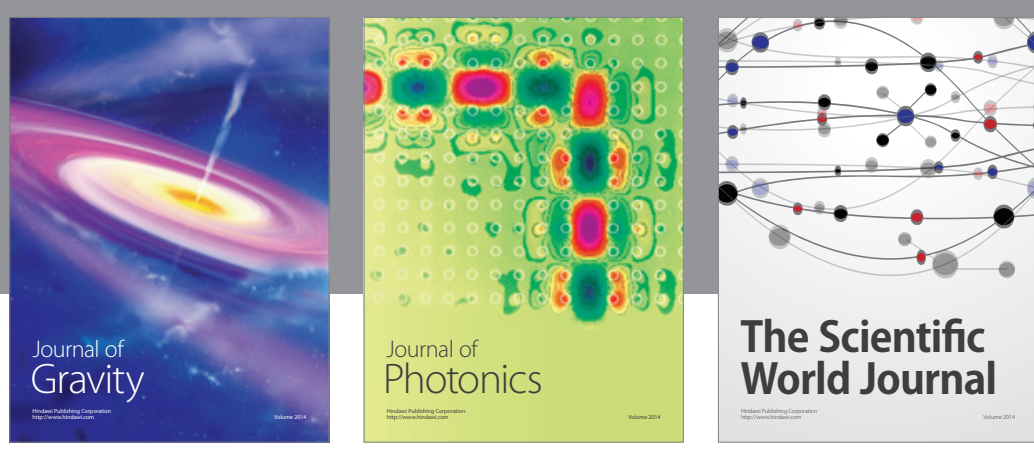

The Scientific World Journal
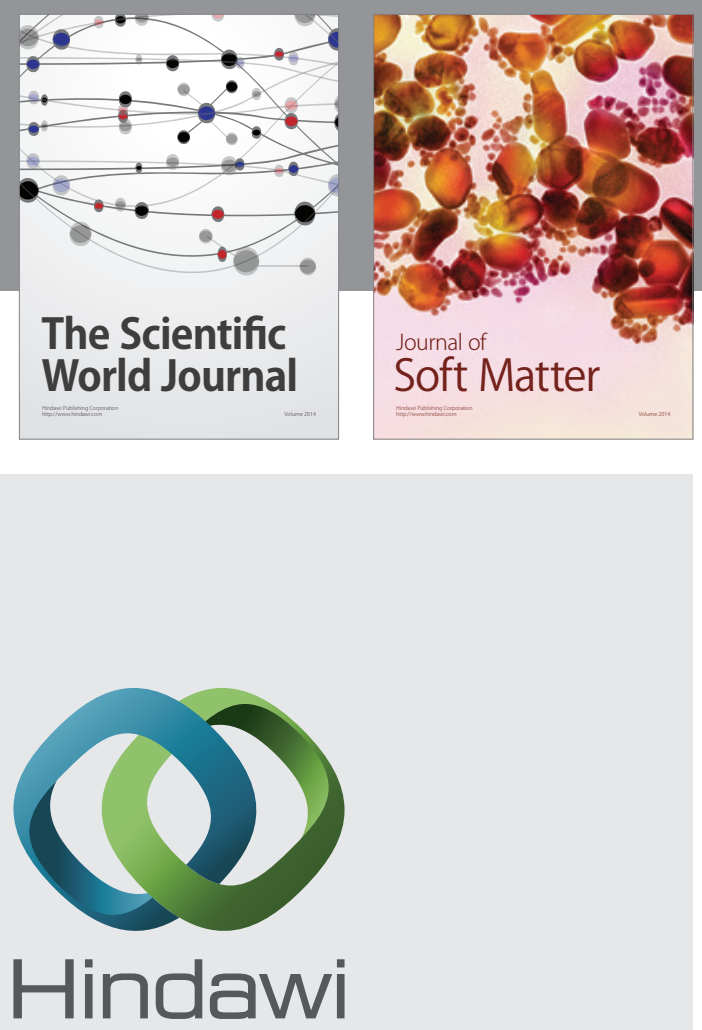

Submit your manuscripts at

http://www.hindawi.com

nternational Journal of

Statistical Mechanics
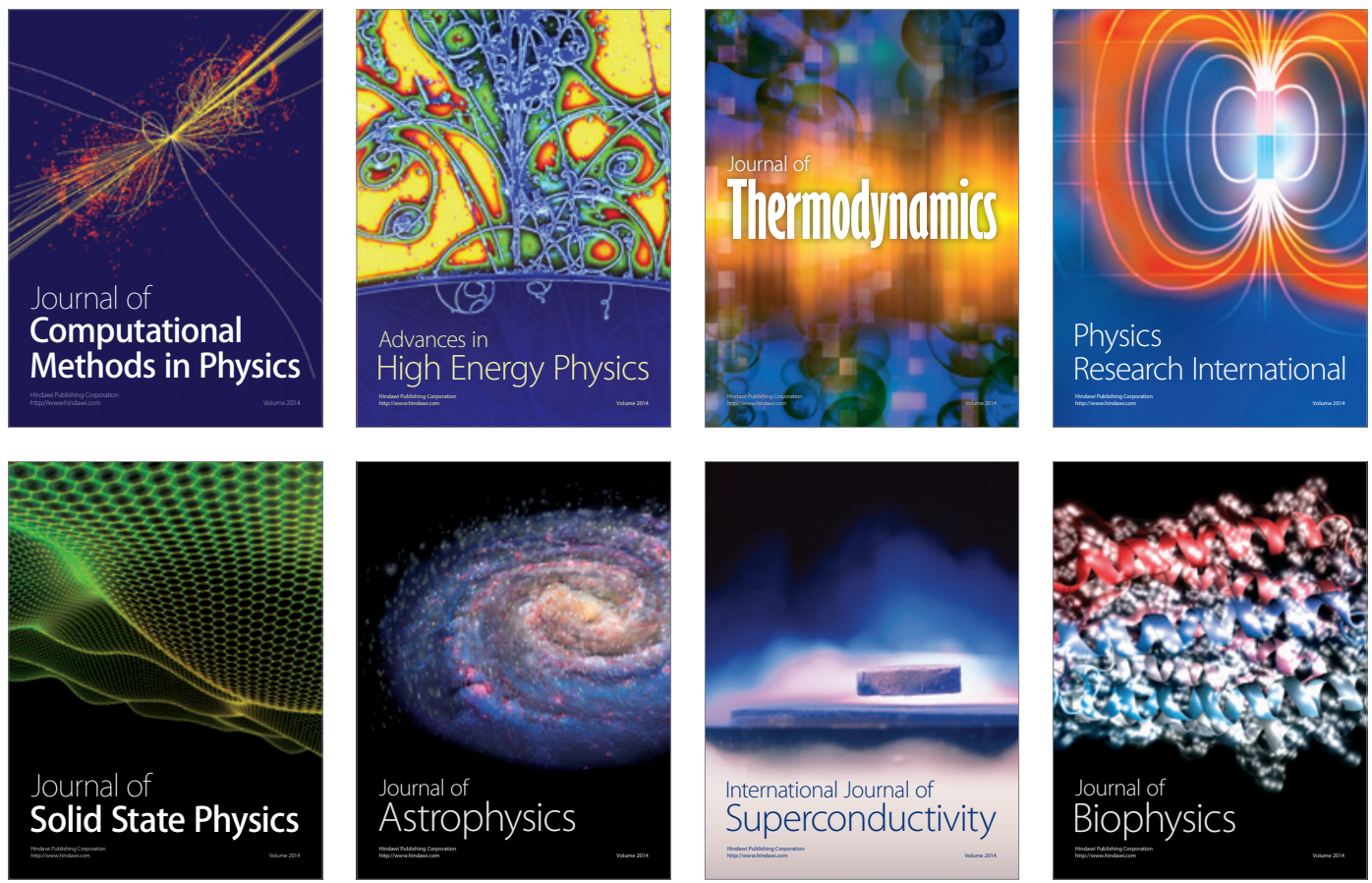
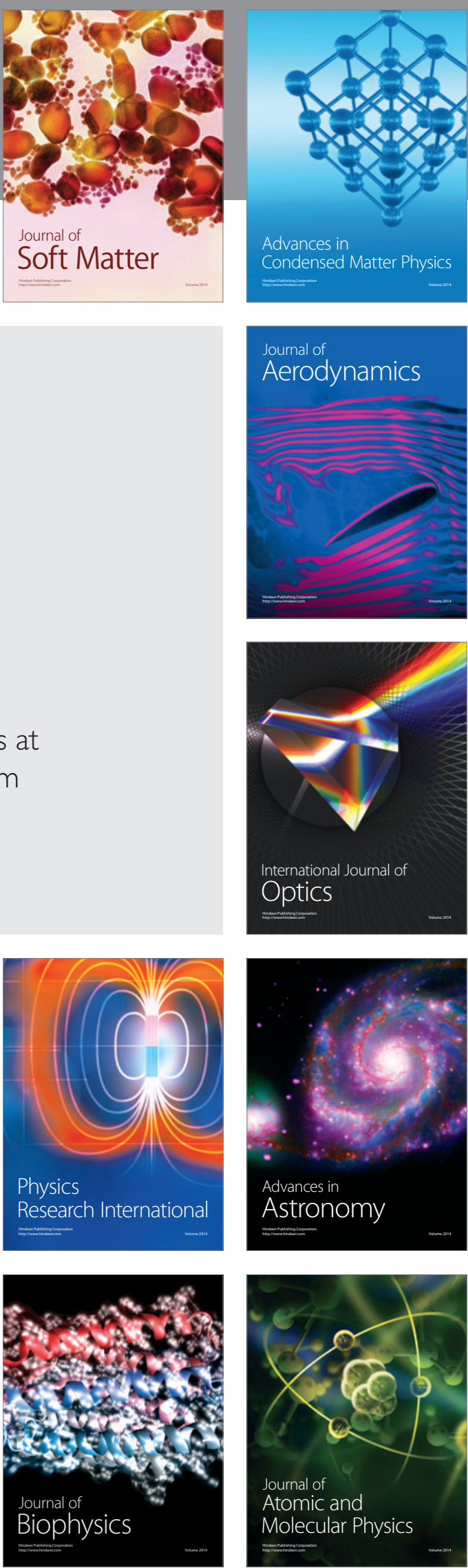